\title{
RLS Impedance Intelligence Control Algorithm for Wire Peeler of Robot in Complex Power Networks
}

\author{
Xianjin Xu $\mathbb{D}^{1},{ }^{1}$ Shichao Hu, ${ }^{1}$ Yu Yan $\mathbb{D}^{\circ},{ }^{2}$ Yuhang Yang $\mathbb{D}^{1},{ }^{1}$ Zhiyong Yang $\mathbb{D}^{1}$, \\ and Haoda Chen (iD ${ }^{1}$ \\ ${ }^{1}$ College of Mechanical Engineering, Hubei University of Technology, Wuhan 430068, China \\ ${ }^{2}$ State Grid of Hunan Electric Power Company Maintenance Company, Changsha 410004, China \\ Correspondence should be addressed to Xianjin Xu; xxjoyjn@126.com
}

Received 20 August 2020; Revised 20 September 2020; Accepted 13 October 2020; Published 29 October 2020

Academic Editor: Ruoli Tang

Copyright ( $\odot 2020$ Xianjin Xu et al. This is an open access article distributed under the Creative Commons Attribution License, which permits unrestricted use, distribution, and reproduction in any medium, provided the original work is properly cited.

Considering the wire core which is easily damaged because of the instability of the power distribution robot during the process of peeling the insulation layer, we have proposed a cutting force tracking control algorithm based on impedance control that is suitable for the end peeling instrument. At present, the task requirement of sudden changes about environment stiffness cannot be accomplished by many impedance control approaches due to the complexity of working environment stiffness about power distribution robot; then, the Recursive Least Square (RLS) method was introduced into the impedance control algorithm to identify the cable insulation layer and cable core stiffness online to achieve accurate and stable tracking of the cutting force. Furthermore, the impedance control of peeling cable insulation layer and the proposed RLS method were simulated and tested contrastively, and the high-voltage cable peeling experiment was performed. The results of simulation and experiment showed that the force control algorithm based on RLS parameter identification still has good force tracking performance during the environment stiffness changes suddenly, and the steady-state error approaches zero, demonstrating the feasibility and effectiveness of the RLS impedance control algorithm, which has important practical significance for improving power distribution efficiency.

\section{Introduction}

The role of the power distribution robot is to peel the cable insulation layer of the transmission wire and guided current wire in the field of power networks in order to fulfill the parallel connection between the guided current wire and the main high-voltage transmission wire. But two situations usually occur when the end effector of the power distribution robot peels the cable insulation layer due to the complexity of the process, one of which is that the cutting force is so heavy that the wire core is damaged directly by the cutter and the other is that the wire insulation layer cannot be completely removed because of insufficient cutting force, both of which will affect the power distribution effect. It is necessary that the enough and accurate cutting force should be provided by the end effector to improve the power distribution work efficiency; some researchers have tried to design a novel peeler structure to overcome the problem of easy damage to the wire core in terms of structural design; however, the problem of easy damage to the wire core cannot be settled because the accurate cutting force cannot be grasped only through depending on the pure mechanical structure. Considering the fact that the problem cannot be solved purely depending on structural design in the working environment of power distribution robot, some control methods are applied to the wire peeling operation link by many later scholars. The embedded control technology was applied to this field in $[1,2]$; it could appropriately reduce the wire core damage rate to a certain extent through combining with embedded control algorithm based on the experiment, but the embedded control algorithm was limited to its low stability and could not be effectively adapted to the working environment of the power distribution robot. The authors in $[3,4]$ have introduced the master-slave control combined with impedance control method into the operation process of power distribution robot to initially achieve compliant 
contact between the end peeler and wire, but the stiffness between the wire core and insulation layer is different whose stiffness parameter cannot be identified by impedance control; especially, when the environment stiffness changes, the stability of impedance control is so poor that the cutting force error biases largely.

As we know, the impedance control was first proposed by the scholar Hogan in [5-8] in order to achieve the purpose of compliance control by adjusting the stiffness parameter of the end effector to obtain the ideal dynamic relationship between position and force, but the control accuracy closely relies on sufficient knowledge on the environment. In real engineering, the cognition of the environment is limited due to the collection of various practical factors, which results in force errors in impedance control; especially, the task requirements cannot be satisfied when the environment stiffness changes suddenly. This paper focuses on the inaccuracy problem of contact force between wire peeler and wire during the peeling process of power distribution robot to propose an impedance force tracking control algorithm based on the Recursive Least Squares (RLS) method of parameter identification, which can achieve stiffness parameter identification between wire core and wire insulation layer to make the cutting force quickly and accurately track the reference force and achieve the compliant peeling of wire insulation and mainly can provide important practical guiding significance for improving the efficiency of power robot.

\section{Impedance Control Strategy on Wire Peeler}

2.1. The Structure Design of End Peeler. Compared with the complicated and bulky structure of end peeler designed by scientific researchers over the past, the peeler designed in this paper is more flexible and compact, its structure is composed of a cable clamping motor, cable clamping block, and cutting blade, as shown in Figure 1, the inner surface of the clamping block is equipped with oblique thread, a cutting blade is mounted on one side, and the clamping motor rotates forward or backward to separate or close the clamping device. Before operation, the cable clamping motor rotates to separate the cable clamping block and align the thread hole with the cable, then, the clamping motor will rotate toward opposite direction to drive the cable clamping block to close when the cable is clamped, the cutting blade synchronously starts cutting in cable insulation layer, and the depth of the blade cutting in the insulation layer is controlled by force sensor. After the wire clamp block clamps the wire, the rotating motor is controlled to run the drive gear counterclockwise, one side of the drive gear is connected to the cable peeling device which is driven to rotate around the center of the gear, and the cable peeling device clamps the wire, making a circular motion around the central axis of the wire to cut the wire insulation layer, relying on the cutting force to realize the axial feed, and converting the output shaft's rotary motion into the perpendicular circumference motion of the peeling device, which can enhance the smoothness of the peeling device.
2.2. The Design of Cutting Force Impedance Controller. The process of contact between the end peeler of the robot and the cable can be regarded as a second-order dynamic relationship which can be described by the following dynamic equation:

$$
M_{t}\left(X-\ddot{X}_{r}\right)+B_{t}\left(\dot{X}-\dot{X}_{r}\right)+K_{t}\left(X-X_{r}\right)=F-F_{r},
$$

where $M_{t}, B_{t}$, and $K_{t}$ are the inertia matrix, damping matrix, and stiffness matrix, respectively, which commonly determine the dynamic characteristic, $X_{r}$ and $X$ are the reference position and actual position of the end peeler, respectively, $\dot{X}_{r}$ and $\dot{X}$ are the expected velocity and actual velocity, $\ddot{X}_{r}$ and $\ddot{X}$ are the expected acceleration and actual acceleration, respectively, and $F_{r}$ and $F$ are the reference contact force and the actual cutting force during the contact between the end peeler and cable. The contact force is zero before the end peeler contacts the wire; this process can achieve position tracking when the end peeler gradually contacts the wire; the impedance controller will generate the correction arguments of position control and combine the desired reference position values to form position control command, which can achieve the purpose of compliant and nondestructive contact between the end peeler and the wire; the entire process can be explained as shown in Figure 2, including the two-subsystem impedance of the end peeler and wire.

The process in which the end peeler is cut into the wire insulation layer can be regarded as a one-dimensional situation, and the force tracking impedance controller can be designed as follows:

$$
m_{t}\left(\ddot{x}-\ddot{x}_{r}\right)+b_{t}\left(\dot{x}-\dot{x}_{r}\right)+k_{t}\left(x-x_{r}\right)=f-f_{r} .
$$

We can obtain the transfer function of impedance control after taking Laplace transform to equation (2) in the frequency domain:

$$
x_{f}=\frac{e_{f}(s)}{m_{t} s^{2}+b_{t} s+k_{t}} .
$$

Equation (3) can be regarded as a low-pass filter; we combine the correction of the position obtained by filtering the force errors with the reference position value to form the position control command:

$$
x_{d}=x_{r}+x_{f} .
$$

Finally, the obtained position control command is converted into the drive voltage of the servo motor through the position controller. As we know, the proportional link can effectively suppress the system deviation and accelerate the system response in PID domain, and the integral link can eliminate the steady-state deviation in [9-12], so the incremental PI control was taken to use in position control and the incremental PI control algorithm is as follows: 

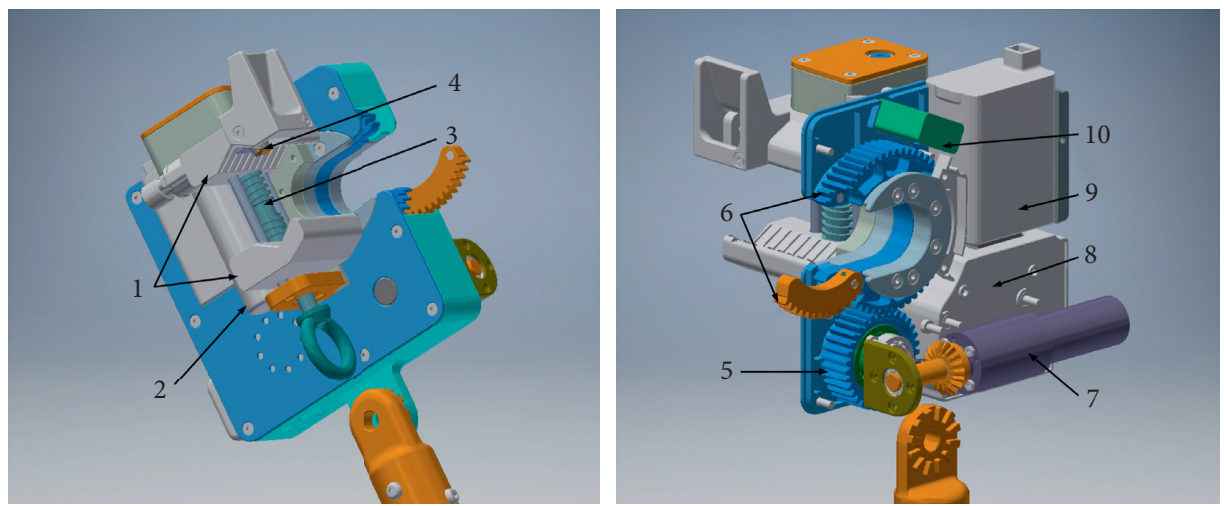

FIGURE 1: Schematic diagram of cable peeling structure. (1) Cable clamping block; (2) cable clamping motor; (3) bidirectional screw; (4) peeling cutter; (5) driving gear; (6) openable gear; (7) rotatory motor; (8) control system; (9) lithium battery; and (10) force sensor.

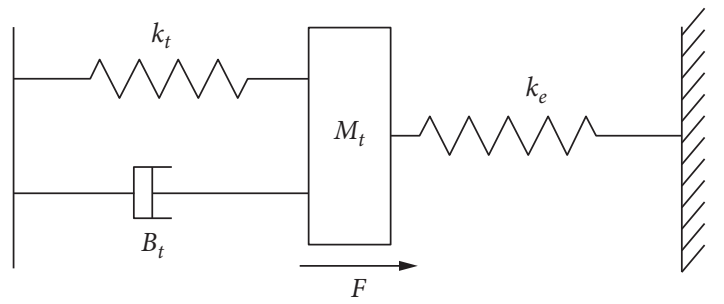

Figure 2: The contact impedance model between cable peeler and wire.

$$
\begin{aligned}
x(k+1) & =x(k)+\Delta x(k+1), \\
\Delta x(k+1) & =k_{p}(e(k+1)-e(k))+k_{i} e(k+1), \\
e(k+1) & =x_{d}(k+1)-x(k+1), \\
x_{d}(k+1) & =x_{r}(k+1)+\Delta x_{f}(k+1) .
\end{aligned}
$$

In the above formula, $x(k)$ and $x(k+1)$ represent the $(k)$ th, $(k+1)$ th position output value, $x(n+1)$ represents the $(n+1)$ th increment, $k_{p}$ and $k_{i}$ are the proportional gain and integral gain, respectively, $x_{r}(k+1)$ is the reference position of the peeler during the $(k+1)$ th sampling, and $x_{d}(k+1)$ represents the desired input value of the position controller.

2.3. The Steady-State Analysis of Impedance System. It is necessary to analyze the stability of the system to improve the force control accuracy of the power distribution robot when the wire insulation layer is being peeled and simultaneously to strengthen the cutting force tracking performance; $f$ is equal to zero in equation (2) before the wire peeler contacts the wire; when the time $t$ approaches $\infty$, then $x$ approaches $x_{r}$, which means that the position tracking is realized, and when the end peeler is in stable contact with the wire, something should be satisfied as follows:

$$
x=x_{e}+\frac{f}{k_{e}} .
$$

Here, $x_{e}$ and $k_{e}$ represent the initial contact position and the stiffness of wire, respectively, and $x_{r}$ represents the reference position value, so

$$
\ddot{x}_{r}=\dot{x}_{r}=0 .
$$

Substituting equation (6) into equation (2), we can obtain the following equation:

$$
\begin{aligned}
m_{t} \ddot{e}_{f}+b_{t} \dot{e}_{f}+\left(k_{t}+k_{e}\right) e_{f}= & m_{t} \ddot{f}_{r}+b_{t} \dot{f}_{r}+k_{t} f_{r} \\
& -k_{t} k_{e}\left(x_{r}-x_{e}\right),
\end{aligned}
$$

where $e_{f}=f_{r}-f$, and when $f_{r}$ is constant, the steady-state deviation can be obtained as

$$
e_{f}(s)=\frac{k_{t}}{k_{e}+k_{t}}\left[f_{r}+k_{e}\left(x_{e}-x_{r}\right)\right]=k_{\mathrm{eq}}\left(\frac{f_{r}}{k_{e}}+x_{e}-x_{r}\right)
$$

where $k_{\text {eq }}$ is the equivalent stiffness of end peeler and wire, which is similar to the series connection of two different stiffness springs, shown as follows:

$$
k_{\mathrm{eq}}=\left(k_{t}^{-1}+k_{e}^{-1}\right)^{-1}=\frac{k_{t} k_{e}}{k_{t}+k_{e}} .
$$

Furthermore, we can obtain the steady-state contact force:

$$
f_{s t}=f_{r}-e_{f}(s)=k_{\mathrm{eq}}\left(\frac{f_{r}}{k_{t}}+x_{r}-x_{e}\right) .
$$

Combining equation (9), we can see that when we know the initial contact position $x_{e}$ and the wire stiffness value $k_{e}$, the force deviation can be made to be zero, in which $k_{e}$ can be estimated by experiment or obtained through experience, and when $x_{e}=x_{r}$, according to equation (2), we can know the natural frequency and damping ratio of the second-order system during the contact between end peeler and wire: 


$$
\begin{gathered}
\omega_{n}=\sqrt{\frac{\left(k_{t}+k_{e}\right)}{m_{t}},} \\
\xi=\frac{b_{t}}{\left(2 \sqrt{\left(k_{t}+k_{e}\right) m_{t}}\right)} .
\end{gathered}
$$

The stability during the contact between the peeler and the wire depends on the target impedance controller parameters, and the dynamic performance depends on the damping ratio and natural frequency. As we know, the greater the inertia is, the greater the impact of the system is, and the greater the damping is, the smaller the system overshoot is, but the system energy consumption will increase. The smaller the stiffness is, the smaller the steady state of force control will be and the system response will slow. The estimation error $\Delta x_{e}$ of $x_{e}$ satisfies the following formula:

$$
-k_{t}^{-1} f_{r}<\Delta x_{e} \leq k_{t}^{-1} f_{r} .
$$

At this time, the desired cutting force is not affected by the position control error, which has strong robustness to the uncertainty of the system model and the disturbance of the contact force.

\section{The Impedance Control Based on RIS Method}

3.1. The Deduction of Recursive Least Square Algorithm. When the end effector of the power distribution robot is in contact with the environment, to accurately measure the contact force, we must rely on full cognizance to the environmental stiffness which cannot be measured directly, so we need to obtain the environment stiffness through an indirect method. The Recursive Least Square (RLS) algorithm is more and more used by virtual of its simple principle, a small amount of calculation, and online parameter identification [13-15], which can be effectively integrated with other control algorithms to improve performance [16], so we can use the iterative characteristics of the RLS algorithm to get the stiffness information of environment and further to get the best control effect approaching to ideal situation. In this paper, the RLS algorithm is introduced into impedance control to perform online identification of wire insulation stiffness and core stiffness, which can effectively improve the force control accuracy of power distribution robots during peeling the insulation.

In Section 2.3, the stable contact force deviation $e_{f}$ (equation (9)) is obtained, and we set the difference between the actual displacement and the assumed displacement to be $\Delta x_{e}$ and the difference between the actual environmental stiffness and the assumed environmental stiffness to be $\Delta k_{e}$; then

$$
\left\{\begin{array}{l}
\Delta x_{e}=x_{e}-\widehat{x}_{e}, \\
\Delta k_{e}=k_{e}-\widehat{k}_{e} .
\end{array}\right.
$$

Further, we can obtain

$$
e_{s}=\frac{k_{t}}{k_{e}+k_{t}}\left(k_{e} \Delta x_{e}-\frac{\Delta k_{e}}{\widehat{k}_{e}} f_{r}\right) \text {. }
$$

Therefore, the main factors that cause force deviation are environment stiffness and position deviation; usually, the position error is not so large that the main factors causing the deviation are environment stiffness during the operation of the power distribution robot. So we can update the reference position $x_{r}$ timely by using the method of online parameter identification based on the RLS algorithm to obtain the wire stiffness information and further to achieve accurate control of the cutting force. Let $\varphi(i), \phi(i)$ be the expected output signal and the actual output signal of the impedance controller, respectively; the signal error can be described as

$$
e(i)=\phi(i)-\varphi(i)
$$

The recursive calculation function can be shown as

$$
\left\{\begin{array}{l}
L(n)=\sum_{i=1}^{n} e^{2}(i)=\sum_{i=1}^{n}[\phi(i)-\varphi(i)]^{2}, \\
e(i)=\phi(i)-Z^{T} X(i),
\end{array}\right.
$$

where $Z$ is the weight function, the forgetting factor $\lambda, \lambda \in(0,1]$ is introduced when the control system is unstable, and the recursive calculation function is synchronously modified as

$$
\begin{aligned}
L(n) & =\sum_{i=1}^{n} \lambda^{n-1} e^{2}(i)=\sum_{i=1}^{n} \lambda^{n-1}[\phi(i)-\varphi(i)]^{2} \\
& =\sum_{i=1}^{n} \lambda^{n-1}\left[\phi(i)-Z^{T} X(i)\right]^{2} .
\end{aligned}
$$

To deploy the above function into a power series,

$L(n)=e^{2}(n)+\lambda e^{2}(n-1)+\lambda^{2} e^{2}(n-2)+\cdots+\lambda^{n-1} e^{2}+\lambda^{n} e^{2}$.

From the above equation, we can find that the coefficient of the quadratic to error is 1 ; suppose that $\lambda$ is so smaller that the energy signal approaches closely the latest quadratic to the error, and the proportion of errors in front of the equation become more and more small, meaning that the parameter identification effect is better. The function of the forgetting factor is to avoid data saturation in the process of obtaining the environmental stiffness parameters [13, 14], and simultaneously, the forgetting factor has been introduced to improve the convergence speed of the algorithm and update the stiffness parameters in time [17, 18], which is beneficial to the reliability of the data. The identified performance generally can be expressed through the minimum variance of the error during the process of parameter identification, and the average errors usually are chosen to be the threshold, when the errors are less than the threshold, meaning that the system gradually approaches stability during this period and the value of forgetting factor $\lambda$ is finitely close to 1 , which can reduce the identification error. 
On the other hand, $\lambda$ is greater than the error threshold, meaning that the system parameters have mutated and $\lambda$ has approached $\lambda_{\min }$ along a curve. Considering that the value range of forgetting factor is $\lambda \in[0,1)$ and in order to accelerate the identification speed, the correction principle of forgetting factor is shown in Figure 3 and can be expressed by the following equation:

$$
\lambda(t)=1-\alpha_{1}\left[\frac{\arctan \left(\alpha_{2}\left(|e(t)|-\alpha_{3}\right)\right)}{\pi+0.5}\right] .
$$

Here, $\alpha_{1}, \alpha_{2}$, and $\alpha_{3}$ have played different roles in the adjustment process of the forgetting factor according to the size of the error; when the error is greater than the threshold $\alpha_{3}$, the value of the forgetting factor is $1-\alpha_{1}$, which is smaller during the period and can accelerate the process of adjustment to reduce the error. When the error is so small that its value infinitely approaches 1 to make the system stable and the whole adjustment process makes the error stable around $\alpha_{2}$ and changes smoothly, the problem of untimely cutting force tracking caused by the excessive variation of the error caused by the sudden change of input signal has been solved according to adjustment of the forgetting factor, and the defect that the ability of parameter correction in RLS becomes weak with the increase of the number of iterations has been settled, which has obvious advantages for the time-varying stiffness parameter identification.

In order to make the identification effect of stiffness parameters better, we need to get the best weight function

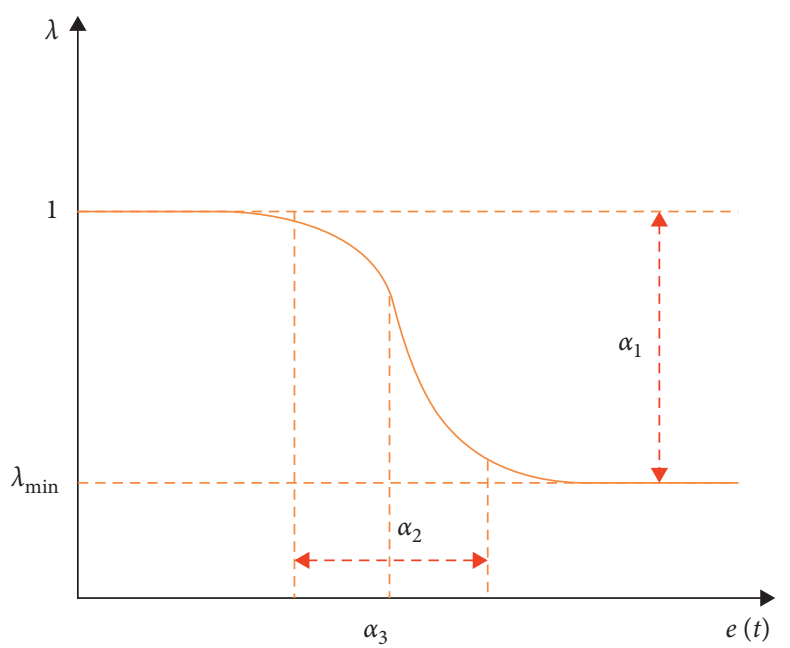

Figure 3: Correction schematic diagram to the forgetting factor.

from theoretical analysis, and if we want to get the best weight function $Z$, we should minimize the iteration function $Z_{k}(n)$, so differentiate equation (21) to obtain

$$
\frac{\partial L(n)}{\partial Z_{k}(n)}=-2 \sum_{i=1}^{n} \lambda^{n-1} e(i) X(i)=0 .
$$

Further, simplify equation (21) to get

$$
\left\{\begin{array}{l}
\sum_{i=1}^{n} \lambda^{n-1}\left[\left[\phi(i)-Z^{T} X(i)\right] X(i)=0\right. \\
Z \sum_{i=1}^{n} \lambda^{n-1} X(i)^{T} X(i)=\sum_{i=1}^{n} \lambda^{n-1} X^{T}(i) \phi(i) .
\end{array}\right.
$$

For calculating conveniently, let us define the following equation:

$$
\left\{\begin{array}{l}
Q(n)=\sum_{i=1}^{n} \lambda^{n-1} X^{T}(i) X(i) \\
P(n)=\sum_{i=1}^{n} \lambda^{n-1} X T(i) \phi(i)
\end{array}\right.
$$

Then equation (22) can be expressed as

$$
Z Q(n)=P(n),
$$

where $Q(n)$ and $P(n)$ are both functions of $n$; we can see that $Z$ is a function of $n$, so there is

$$
Z(n)=Q^{-1}(n) P(n)=R(n) P(n) .
$$

Here, $R(n)=Q^{-1}(n)$, and equation (23) can be denoted as the following iterative form:

$$
\left\{\begin{array}{l}
Q(n)=\lambda Q(n-1)+X(n) X^{T}(n), \\
P(n)=\lambda P(n-1)+\phi(n) X(n) .
\end{array}\right.
$$

Substitute $R(n)$ into equation (26) to get

$$
\begin{aligned}
R(n) & =\left[\lambda R^{-1}(n-1)+X(n) X^{T}(n)\right]^{-1} \\
& =\frac{1}{\lambda}\left[R(n-1)-K(n) X^{T}(n) R(n-1)\right] .
\end{aligned}
$$

Define the gain $K(n)$ as follows:

$$
K(n)=\frac{R(n-1) X(n)}{\lambda+X^{T}(n) R(n-1) X(n)} .
$$

Substitute equations (28) and (26) into equation (25) to get the final expression of weight function:

$$
Z(n)=Z(n-1)+K(n) e(n-1) .
$$

The entire RLS algorithm can be represented as Figure 4 according to the flowchart.

3.2. The Impedance Control Based on RLS Algorithm. The process of contact between the end peeler and the wire is 


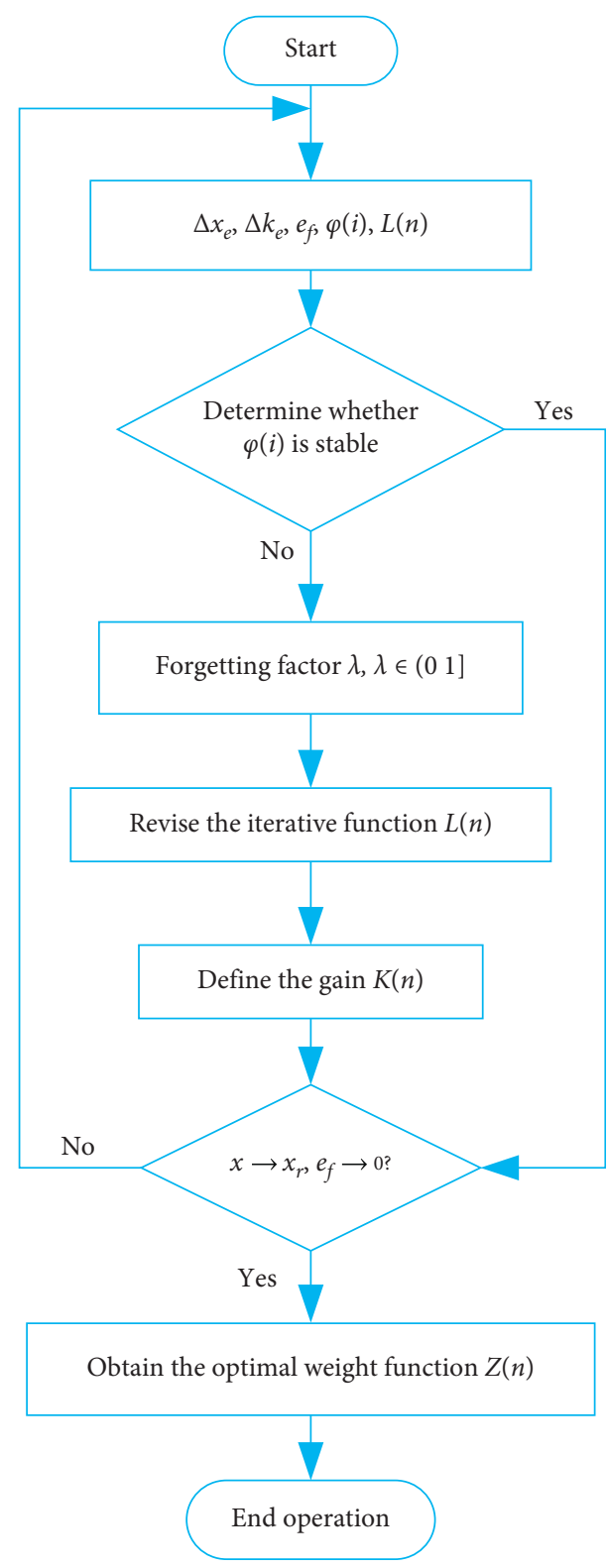

FIgUre 4: The flowchart of RLS algorithm.

regarded as the action borne force of the spring with stiffness $k_{e}$, which can be regarded as a one-dimensional situation where $f=k_{e} x$, and $f$ is the actual cutting force applied to the wire by the end peeler and being collected by the force sensor, $x$ is the displacement of the end peeler which can be measured by the motor encoder, any set of data $\left(x_{t}, f_{t}\right)$ is obtained during the sampling process, and $\widehat{k}_{e}$ is estimated by the RLS algorithm.

So the following relation can be gained:

$$
\begin{aligned}
\widehat{k}_{e}(t) & =\left(x_{t}^{T} x_{t}\right)^{-1} x_{t}^{T} f_{t}, \quad\left(t \in N^{*}\right), \\
R_{t+1} & =\frac{R_{t}}{\left(\lambda_{t}+x_{t+1}^{T} x_{t+1}\right)}, \\
\widehat{K}_{e}(t+1) & =\widehat{K}_{e}(t)+K_{e}(t+1)\left(f_{t+1}-\widehat{k}_{e}(t) x_{t+1}\right), \\
k_{e}(t+1) & =R_{t+1} x_{t+1} .
\end{aligned}
$$

The RLS algorithm is applied to the wire peeling process of the power distribution robot; in order to reduce the data error, the average value obtained during the sampling period is used to be the actual value of the cutting force to the wire peeler. When the errors where the values are compared with expected reference force to get are less than 0.01 , the RLS impedance system can be regarded as stable, the calculation based on the Recursive Least Square method is being stopped, and the updating algorithm of reference position to the cutting force based on RLS parameter identification can be obtained:

$$
\begin{aligned}
& x_{r}(t+1)=\frac{f_{r}}{k_{e}(t+1)}+x_{e}, \\
& x_{d}(t+1)=\frac{f_{r}}{k_{e}(t+1)}+x_{e}+x_{f} .
\end{aligned}
$$

The entire principle of impedance control process based on RLS algorithm can be described by the following impedance control system diagram frame shown in Figure 5.

\section{Simulation Analysis}

The power distribution robot is taken to be research object in this paper to consider the problem of the unstable cutting force which is produced by the end peeler and the wire during the peeling process, and we have proposed an impedance control algorithm based on Recursive Least Square (RLS) method of parameter identification to achieve a smooth contact between the peeler and wire. To verify whether the algorithm can achieve stable tracking of the cutting force in the case of sudden changes to environmental stiffness, the algorithm of each controller is written and sealed in the Simulink environment.

4.1. Simulation of Impedance Control. In order to make the response speed of each controller the best and the system overshoot and the steady-state error the lowest, we have run and debugged the simulation system many times, and the parameters are set to be $m_{t}=1, b_{t}=120 \mathrm{~N} /(\mathrm{m} / \mathrm{s}), k_{t}=$ $800 \mathrm{~N} / \mathrm{m}, k_{p}=4.07$, and $k_{i}=2.38$, respectively, which can refer to the effective literature $[19,20]$, the stiffness of wire to be $k_{e}=1000 \mathrm{~N} / \mathrm{m}$, and the equivalent stiffness of system to be $k_{\text {eq }}=444 \mathrm{~N} / \mathrm{m}$. Considering that the wire insulation layer is generally plastic and the wire core is metal whose stiffness is different, when carrying out impedance simulation, we perform the tracking simulation including the constant force 


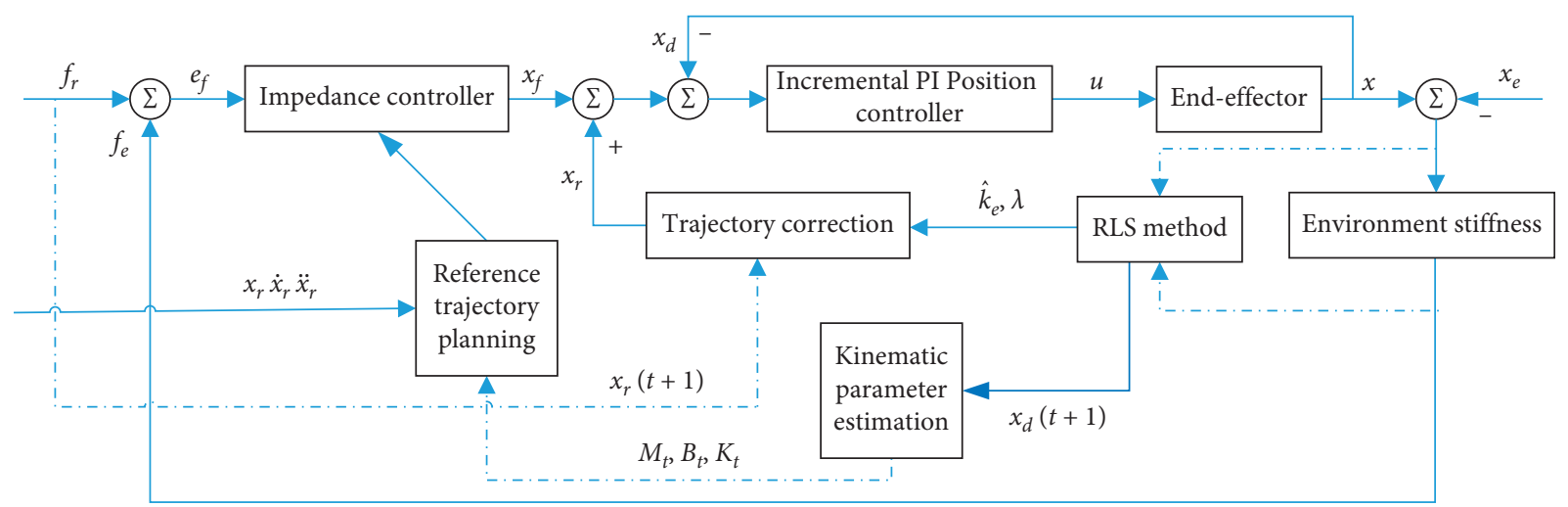

FIgURE 5: The block diagram of RLS impedance control system.

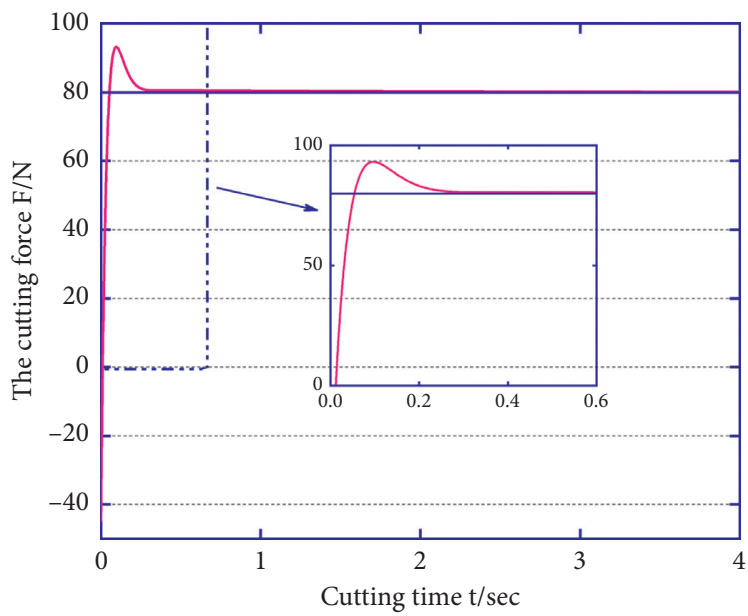

_ Impedance control force

— Reference force

FIgURE 6: The response curve of constant force tracking.

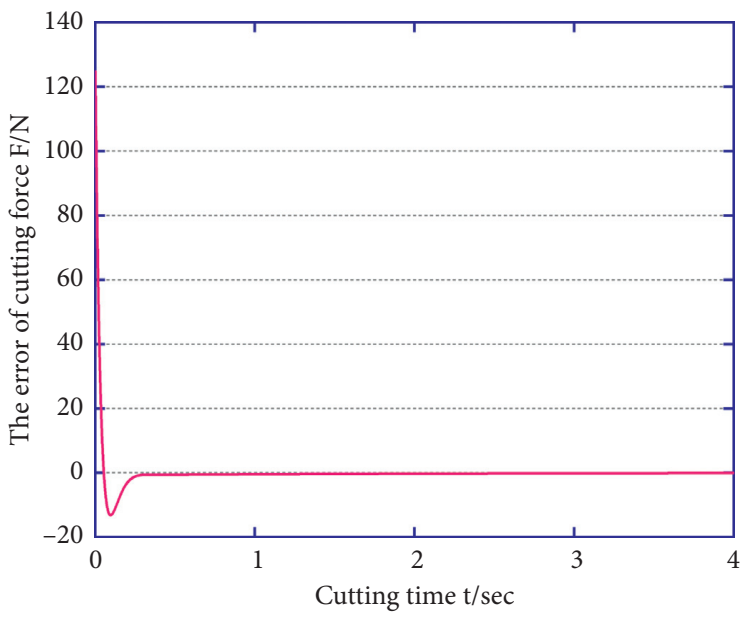

_ The force error of impedance control

FIgURE 7: The error curve of constant force tracking. 


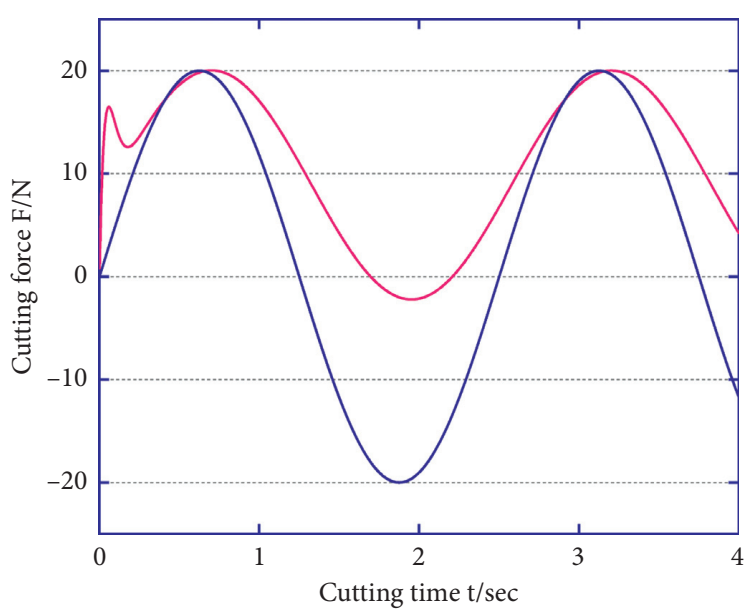

- Impedance control force

- Reference force

FIgURE 8: The response curve of sine force tracking.

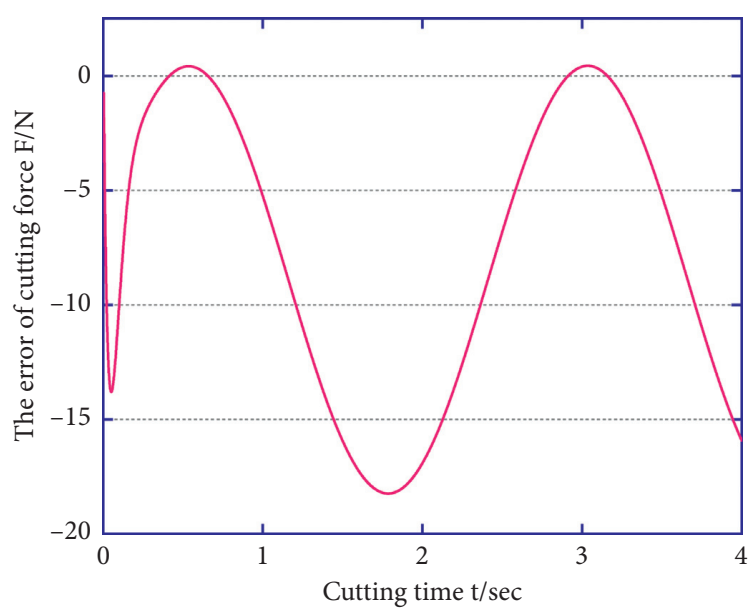

_ Error of impedance control

Figure 9: The error curve of sine force tracking.

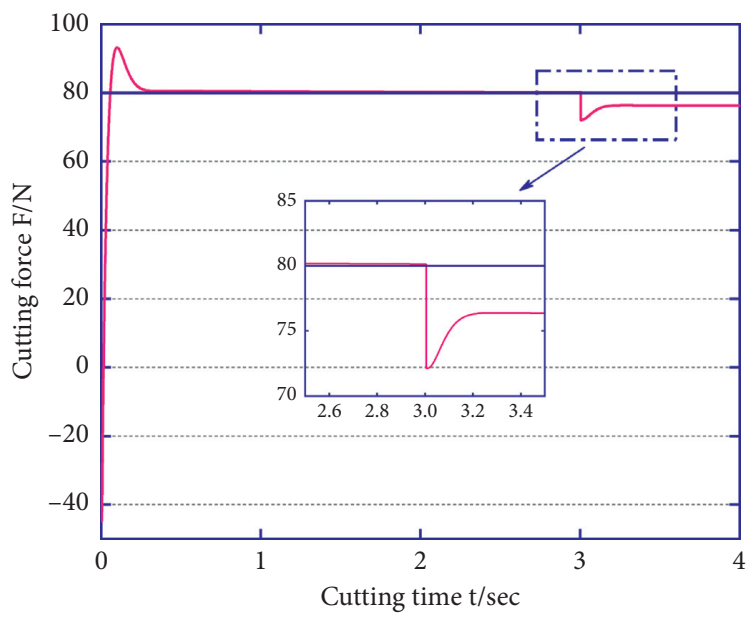

The force of impedance control

— Reference force

FIGURE 10: The constant force tracking curve on mutated stiffness. 


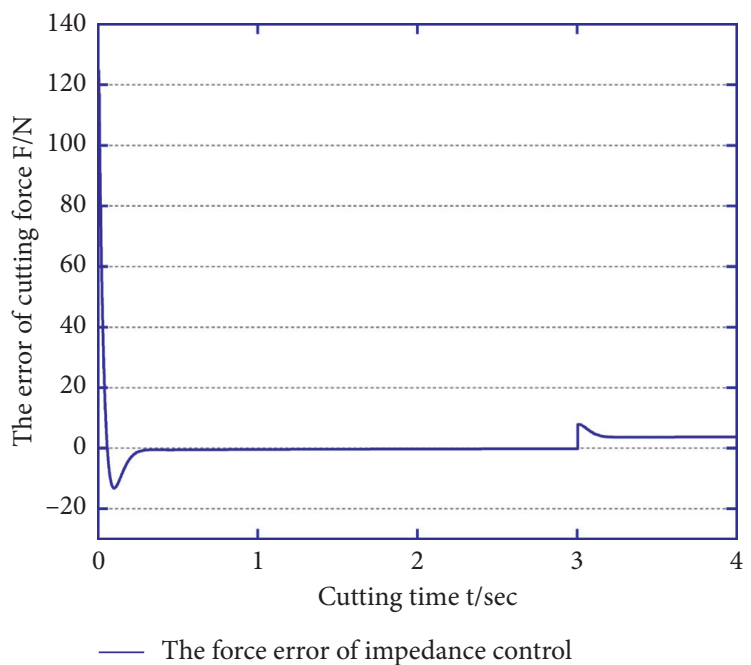

FIgURE 11: The curve of constant force tracking on mutated stiffness.

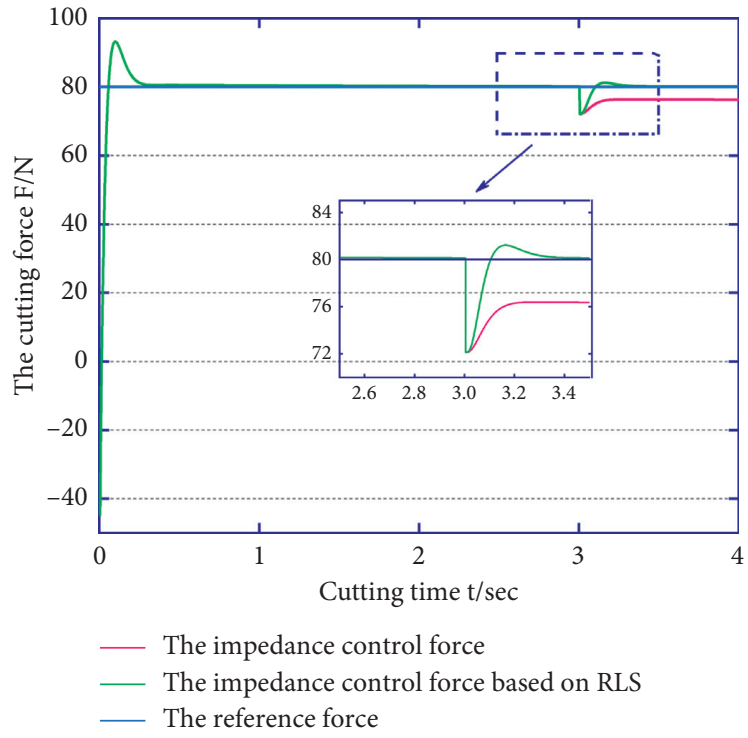

FIGURE 12: The constant force tracking response curve of RLS on mutated stiffness.

$F=80 \mathrm{~N}$ and the sine force $F=20 \sin (0.8 \pi)$ whose amplitude is 20 and frequency is $0.4 \mathrm{~Hz}$, respectively, assuming that the environment stiffness mutates suddenly after simulating $3 \mathrm{~s}$ and the mutated environment stiffness is $k_{e}^{\prime}=900 \mathrm{~N} / \mathrm{m}$, setting the total simulation time to be $4 \mathrm{~s}$, the force sampling time to be $1 \mathrm{~mm}$, and the initial position of the peeler to be $0.05 \mathrm{~mm}$; the simulation results can be obtained and shown as in Figures 6-11.

4.2. The Impedance Control Based on RLS Algorithm. In order to verify that the RLS algorithm still has good force tracking performance when the environment stiffness changes suddenly, the impedance control algorithm of the power distribution robot is comparatively simulated under the same conditions, the parameters of each controller are set to be the same as in Section 3.1, the forgetting factor which belongs to Recursive Least Square method is set to be $\lambda=0.975$, and the following simulation results are obtained as shown in Figures 12-17.

It can be seen from the simulation results that the impedance control based on RLS can respond quickly and stably in a relatively short period of time before and after the environmental stiffness mutates, and the error is always approaching zero whether it is a constant force tracking or a sine force tracking, which should attribute 


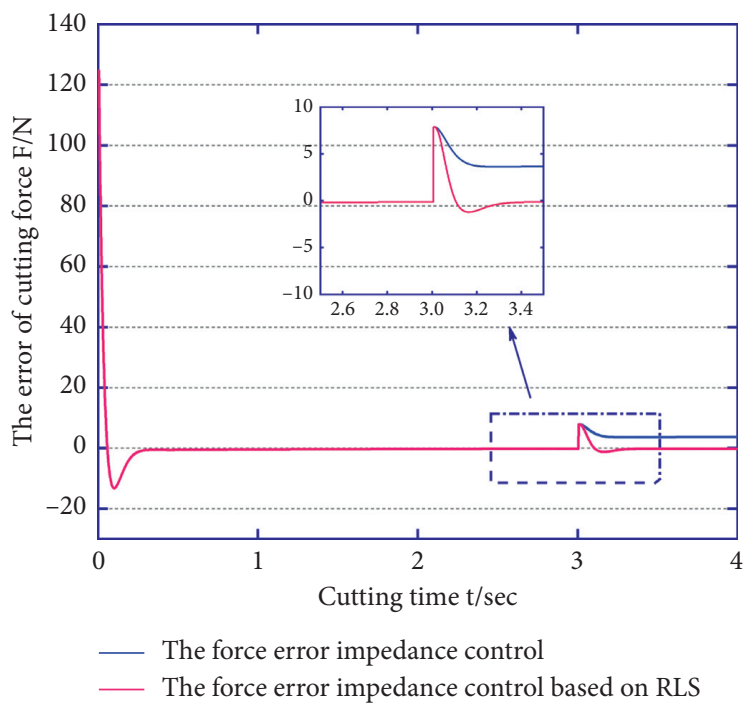

FIgURE 13: The constant force tracking error curve of RLS on mutated stiffness.

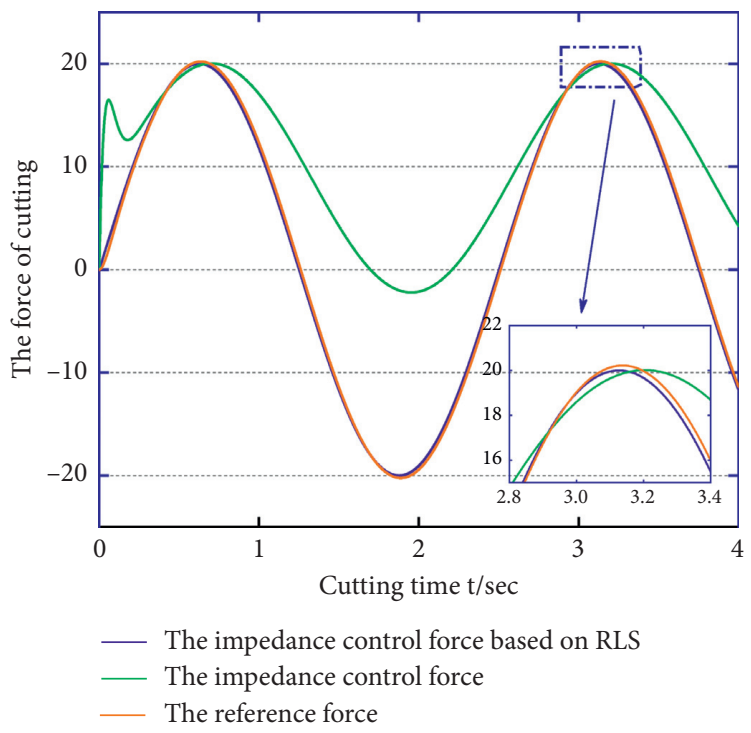

FIGURE 14: The response curve of sine force based on RLS.

to the superiority of the RLS impedance control algorithm to a certain extent; it can reflect the feasibility and effectiveness of the impedance control algorithm based on RLS, compared with the impedance control algorithm of the peeling link in the power distribution robot operation. On the contrary, compared with the simulation results of impedance control in Figures 10 and 11, we can know that impedance control can only complete certain tasks without any mutated environmental stiffness, but it is difficult to complete tasks when the environmental stiffness changes suddenly that is because ordinary impedance control cannot identify the environmental stiffness.

\section{Experiment}

When performing the peeling experiment of the robot's high-voltage wire, we firstly select the commonly used wire whose main core is steel and the subordinate material is an aluminum strand in the $10 \mathrm{kV}$ distribution networks (GB/ T1179-2008: round and overhead wire with concentric strand), and the performance parameters of the high-voltage are shown in Table 1.

The experimental site is shown in Figure 18, and the end peeler has been assembled with the end flange of the UR10e, which is a collaborative 6-axis robot to verify the 


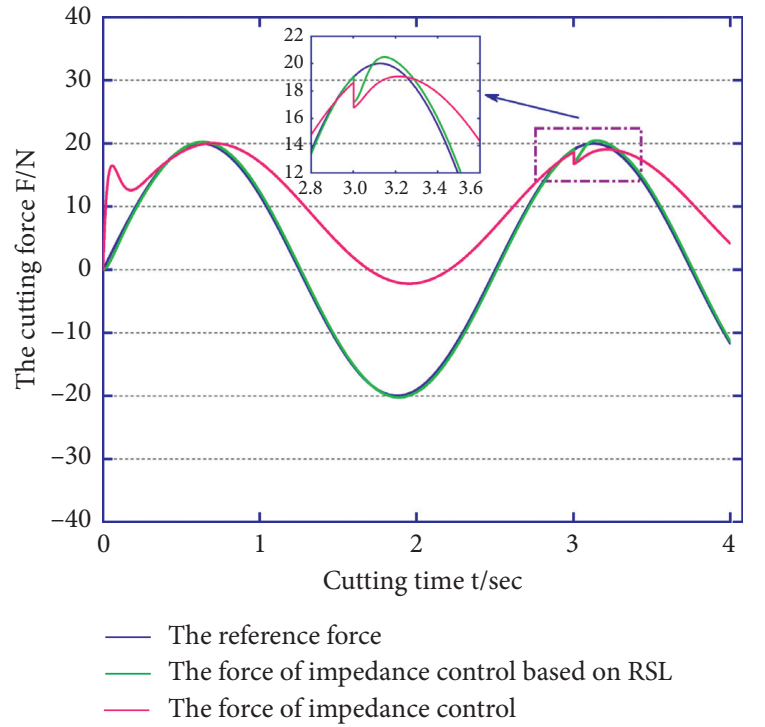

FIgURE 15: The sine force tracking response curve based on RLS of mutated stiffness.

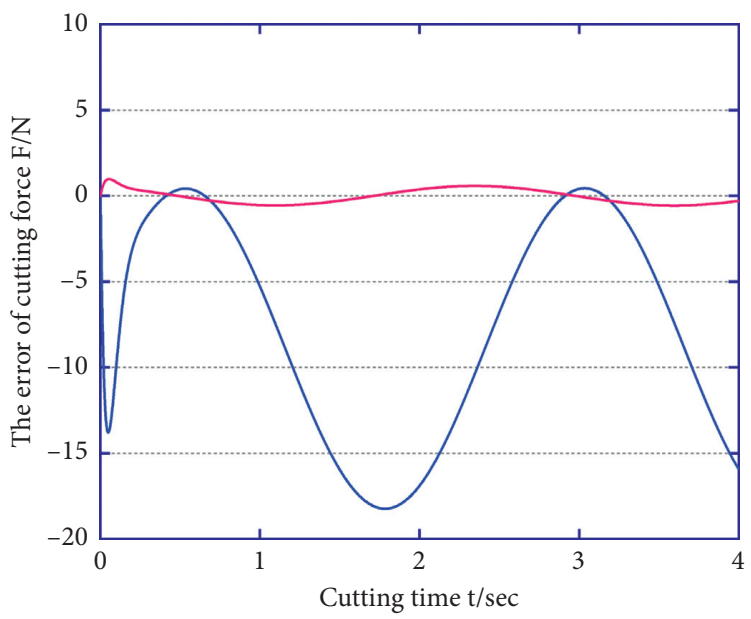

_ The error of impedance control

— The error of impedance control based on RLS

Figure 16: The sine force tracking error curve of RLS.

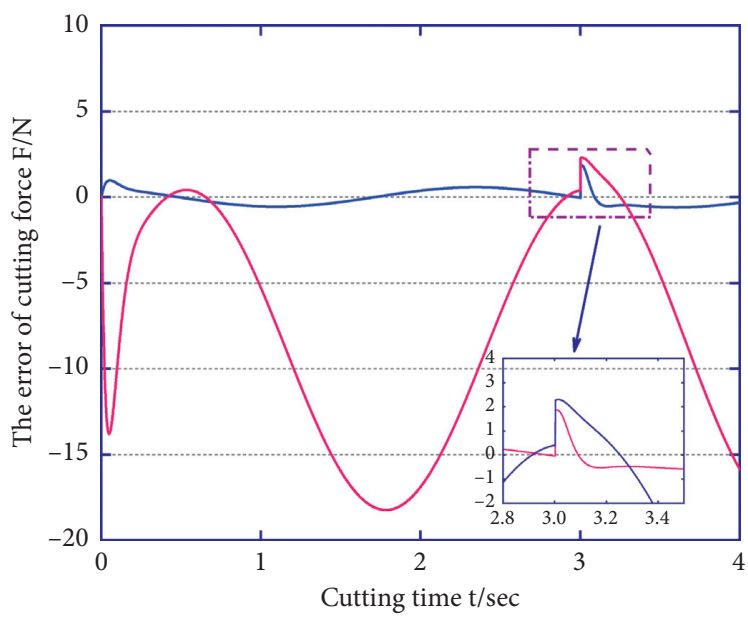

The error of impedance control based on RLS

The error of impedance control

FIGURE 17: The sine force tracking error curve of RLS on mutated stiffness. 
TABLE 1: The performance parameter of high-voltage wire.

\begin{tabular}{lcc}
\hline $\begin{array}{l}\text { Normal cross-section (aluminum/steel) } \\
\text { Steel proportion }\end{array}$ & $150 / 20$ \\
Number of single wires & Aluminum & $13 \%$ \\
Standard thickness of insulation $(\mathrm{mm})$ & Steel & 24 \\
Diameter $(\mathrm{mm})$ & Steel core & 3.4 \\
The insulation material & Helix line & 5.55 \\
Density of XLPE $\left(\mathrm{g} / \mathrm{cm}^{3}\right)$ & & 16.7 \\
Elastic modulus of XLPE $(\mathrm{MPa})$ & & 0.92 \\
\hline
\end{tabular}

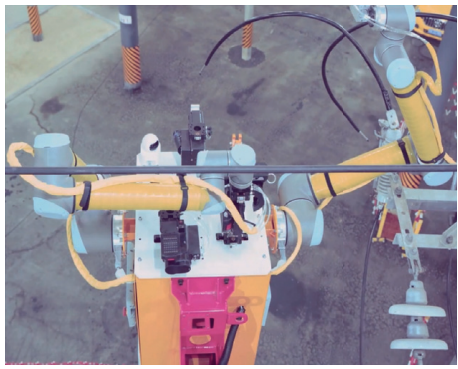

(a)

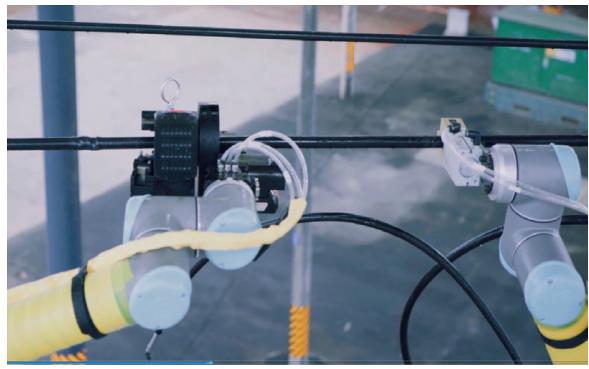

(b)

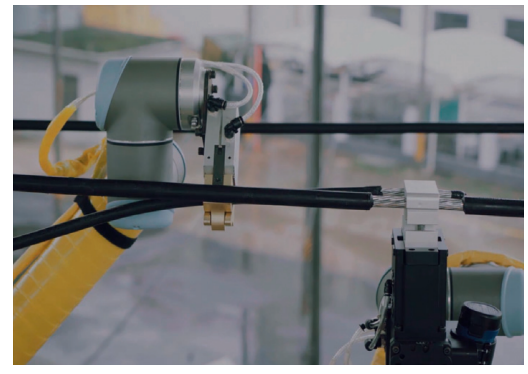

(c)

Figure 18: The RLS impedance control experiment to peeler of power robot. (a) Preparation process before peeling. (b) Process of peeling insulation. (c) Connect the lead current wire.

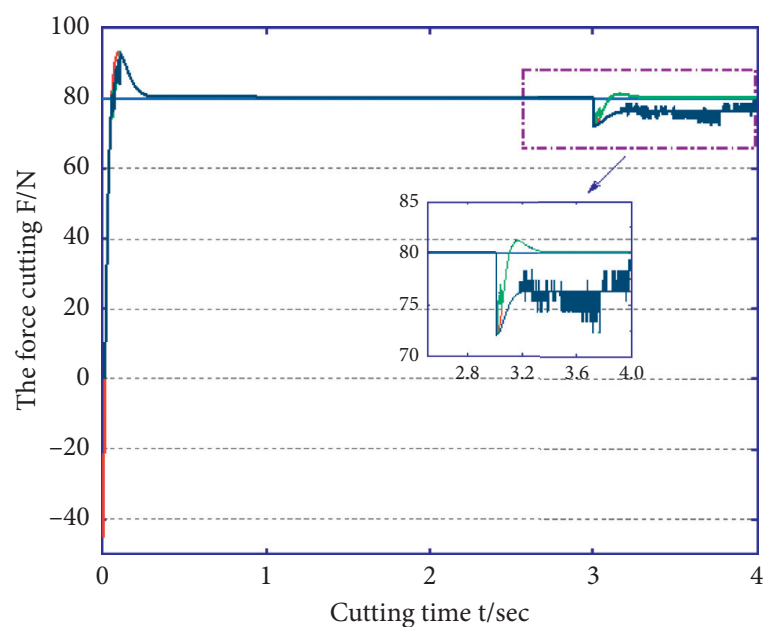

— The reference force

_ The simulation force based on RLS algorithm

- The simulation force of impedance control

- The experimental value based on RLS algorithm

_ The experimental value based on impedance control

(a)

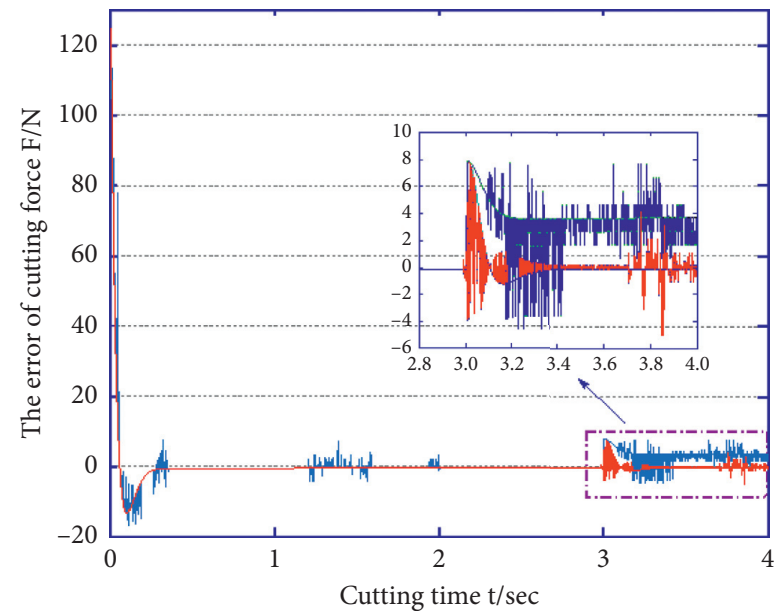

- The simulated force error of impedance control

_ The simulated force error of impedance control based on RLS algorithm

_ The experimental value to impedance control based on RLS algorithm

— The experimental value to impedance control

(b)

FIGURE 19: Comparison of the simulated and experimental values.

peeling effect of the cutting force control algorithm to the peeler during the peeling process of the power distribution networks. In the process of setting the force controller parameters, the sampling interval of force control is set to be $T_{s}=1 \mathrm{~ms}$, the time that is used to peel the wire insulation layer is set to be $4 \mathrm{~s}$, the speed of the wire peeler which moves around the wire axial is $3.75 \mathrm{~cm} / \mathrm{s}$, the parameters to the target impedance 
controller are set to be the same as the previous chapter during peeling the wire, and the test of impedance control and the test being improved RLS impedance control are carried out, respectively.

5.1. Analysis of Experiment. Comparing the experimental values with simulated values in Figure 19, it can be seen that they are basically identical, and what is most important is that the errors of cutting force, experimental and simulated, especially the force value to impedance control based on RLS algorithm, are so small, approaching zero, which proves that the proposed strategy to impedance control based on RLS algorithm is accurate and feasible; last but not least, the wire can be peeled clearly and not be damaged during the robot peeling experiment, which has important practical significance for improving power distribution efficiency and the development of power distribution networks.

\section{Conclusion}

Considering the wire core which is easily damaged due to the instability of the power distribution robot in the field of distribution networks operations, we have proposed an impedance force tracking control algorithm based on RLS algorithm, which can effectively identify the stiffness between the insulation layer of wire and wire core during the peeling process and can provide more accurate cutting force for the end peeler.

In order to verify the effectiveness and feasibility of the proposed algorithm, a simulation model based on the RLS impedance tracking control algorithm was built in the Simulink environment. At the same time, both the simulation and experiment on peeling link to the impedance control of the power distribution robot and the proposed impedance control algorithm based on RLS were implemented; comparing the results of simulation and experiment, which showed that the proposed RSL algorithm can still have better force tracking performance in the case of a sudden change to environment stiffness and the steady-state error approached zero, this algorithm based on RLS has high stability and feasibility compared with the traditional impedance control, which can provide theoretical guide and important practical significance for solving the problem that the wire core is easily damaged during the operation of power distribution robot. The impedance control algorithm based on RLS parameter identification will be further optimized and improved in the future; this is a difficult and long-term process.

\section{Nomenclature}

RLS: The Recursive Least Square method

$M_{t}\left(m_{t}\right)$ : The inertia matrix of system

$B_{t}\left(b_{t}\right)$ : The damping matrix of system
$K_{t}\left(k_{t}\right): \quad$ The stiffness matrix of system

$\ddot{X}_{r}\left(\ddot{x}_{r}\right)$ : The desired acceleration of peeler

$\dot{X}_{r}\left(\dot{x}_{r}\right)$ : The desired velocity of peeler

$\ddot{X}_{r}\left(\ddot{x}_{r}\right)$ : The desired displacement of peeler

$F(f)$ : The contact force of end peeler

$F_{\mathrm{r}}\left(f_{\mathrm{r}}\right)$ : The reference force of controller

$f_{\mathrm{st}}$ : The steady-state contact force

$x_{\mathrm{r}}: \quad$ The reference position

$x_{e}: \quad$ The initial position of peeler

$x_{d}$ : The control command of position

$k_{e}$ : The stiffness of wire

$k_{\text {eq }}$ : The equivalent stiffness of system

$\xi$ : The damping ratio of system

$\omega_{n}$ : The natural frequency of system

$\alpha: \quad$ The threshold of parameter $\lambda$

$\lambda: \quad$ The forgetting factor

$L(n): \quad$ The recursive calculation function

$Z_{k}(n)$ : The optimal weight function.

\section{Data Availability}

The experiment data used to support the findings of this study are included within the article.

\section{Conflicts of Interest}

The authors declare that there are no conflicts of interest regarding the publication of this paper.

\section{Acknowledgments}

The authors state that the research and publication of this paper were supported absolutely by the National Natural Science Foundation of China (Grant no. 61375092).

\section{References}

[1] J. Li and D. M. Li, "Development of automatic wire stripper for high voltage live working robot," Manufacturing Automation, vol. 15, no. 4, pp. 111-114, 2011.

[2] Y. L. Zhao, H. Qi, F. M. Chen et al., "Development of a special remote wire stripper for high voltage live working robot," Manufacturing Automation, vol. 26, no. 2, pp. 117-119, 2010.

[3] X. L. Ma, Design and Optimization of Working Manipulator of High Voltage Live Working Robot, Shangdong Jianzhu University, Jinan, China, 2012.

[4] S. Y. Lu and M. C. Fu, "DWR-I remote operation high voltage live working robot," Journal of Shanghai Jiaotong University, vol. 6, no. 6, pp. 911-913, 2015.

[5] J. Li, Q. Wei, W. S. Chang et al., "Adaptive force impedance control algorithm based on impedance control," Robot, vol. 1, no. 1, pp. 911-913, 1991.

[6] N. Hogan, "Impedance control: an approach to manipulation: Part I-theory," Journal of Dynamic Systems, Measurement, and Control, vol. 107, no. 1, pp. 1-7, 1985.

[7] T. Lasky and T. Hsia, "On force-tracking impedance control of robot manipulator," in Proceeding of the IEEE International 
Conference on Robotics and Automation, pp. 274-280, Sacramento, CA, USA, April 1991.

[8] A. Farhad, "Robust impedance control of manipulators carrying a heavy payload," Journal of Dynamic Systems, Measurement, and Control, vol. 11, no. 6, pp. 1-8, 2010.

[9] T. N. Yogyakarta, "Efficient PID controller based hexapod wall following robot," in Proceedings of the International Conference on Electrical Engineering, Computer Science and Informatics, Bandung, Indonesia, September 2019.

[10] C. Igncaio, D. P. Mariano, and G. A. Gerardo, "Double Q-PID algorithm for mobile robot control," Expert System with Application, vol. 137, no. 12, pp. 292-307, 2019.

[11] C. F. Juang and C. Hsu, "Reinforcement ant optimized fuzzy controller for mobile-robot wall-following control," in Proceedings of the IEEE Trans. Ind. Electron, Japan, December 2019.

[12] Y. L. Kiam, A. Heong, and C. Gregory, "PID control system Analysis," IEEE Transactions on Control Systems Technology, vol. 13, no. 4, pp. 141-148, 2007.

[13] H. Qian, C. Jiang, Y. K. Pan et al., "Online identification of voltage regulator model based on adaptive forgetting factor RLS algorithm," Nuclear Power Engineering, vol. 6, no. 12, pp. 77-80, 2019.

[14] H. Y. Wang, Y. S. Zhang, and G. Zhang, "Impedance on system identification and adaptive control system simulation algorithm," Control Engineering of China, vol. 15, no. 9, pp. 77-80, 2008.

[15] Q. Q. Luo, J. H. Su, Z. G. Lin et al., "Parameter identification method of virtual synchronous generator based on recursive least square method," Automation of Electric System, vol. 43, no. 9, pp. 215-220, 2019.

[16] H. B. Li, P. Liu, Q. P. Ren et al., "A self-tuning controller of generalized minimum variance with improved Least-squares algorithm under ASM test," Machinery Design \& Manufacture, vol. 6, no. 7, pp. 171-173, 2012.

[17] L. Li, Z. Chen, B. W. Wang et al., "Sensorless robot end-effect contact force estimation based on VFF-RLS," Machinery Design \& Manufacture, vol. 15, no. 9, pp. 77-80, 2017.

[18] H. X. Shen and B. L. Jin, "Permanent magnet synchronous motor forgetting factor recursive least square parameter identification," Journal of System Simulation, vol. 9, no. 9, pp. 3404-3410, 2018.

[19] Z. Yang, "Research on parameter regulation of robot impedance control algorithm," Journal of Zaozhuang University, vol. 25, no. 2, pp. 89-93, 2008.

[20] M. Kushwah and P. A. Patra, "Tuning PID controller for speed control of DC motor using soft computing techniques-a review," Advanced Electronic and Electrical Engineering, vol. 4, no. 2, pp. 141-148, 2014. 